Discrete Comput Geom 28:389-403 (2002)

DOI: $10.1007 / \mathrm{s} 00454-002-0747-6$

\title{
On Extremal Finite Packings
}

\author{
Achill Schürmann \\ Mathematics Department, University of Siegen, \\ 57068 Siegen, Germany \\ achill@math.uni-siegen.de
}

Dedicated to Jörg M. Wills on the occasion of his 65th birthday

\begin{abstract}
We show that in contrast to the classical infinite packing problem, even in the Euclidian plane, the solutions to several finite packing problems are non-lattice packings if the number of translates is large enough. This answers, in particular, a question by Paul Erdös [E].
\end{abstract}

\section{Introduction}

Let $\mathbb{E}^{d}$ denote the Euclidian $d$-space equipped with the norm $\|\cdot\|$, and let $\mathcal{K}^{d}$ be the family of strictly convex, compact convex bodies with non-empty interior in $\mathbb{E}^{d}$. We say a discrete set $X \subset \mathbb{E}^{d}$ defines a packing or $K$-packing $X+K$ if and only if the translates of $K$ have mutually disjoint interiors. We speak of a finite packing if $X=X_{n}=\left\{x_{1}, \ldots, x_{n}\right\}$ is finite, and of a lattice packing if $X$ is subset of a packing lattice $\Lambda$ that is a discrete subgroup of $\mathbb{E}^{d}$ with $\Lambda+K$ being a packing. There are many well-studied problems concerning infinite as well as finite packings, in particular, if $K$ is a sphere (see [CS] and $[\mathrm{Z}])$.

The classical task in the Geometry of Numbers is to find (infinite) packings with maximum density where the density is, loosely spoken, given by the proportion of space covered by the packing (see [GL] for an exact definition). In $\mathbb{E}^{3}$ the Kepler problem and its solution by Hales (see $[\mathrm{H}]$ and $[\mathrm{L}]$ ) show the difficulty of the problem. Here, as in $\mathbb{E}^{2}$ for general $K \in \mathcal{K}^{2}$ (see [F]), maximum density is attained by a lattice packing $X+K$. It is an open problem if there exists a $K \in \mathcal{K}^{d}$ for $d \geq 3$, for which the maximum density is not attained by a lattice packing.

In this paper we show that solutions $X_{n}+K$ to several finite packing problems, already in $\mathbb{E}^{2}$, are always non-lattice packings if $n$ is large enough. For this we consider a class of 
packing problems for a given $K \in \mathcal{K}^{2}$, where the quality of a packing is determined by a "packing function" (see Definition 2). This includes in particular (see [BF], [BHW], $[\mathrm{CFG}],[\mathrm{GW}]$, and $[\mathrm{F}]$ ) the following well-known problems:

Problem 1 (Maximum Parametric Density Problem). Determine packings $X_{n}+K$ with minimum area $\mathcal{A}\left(\operatorname{conv}\left(X_{n}+K\right)\right)$ of their convex hull or, more general, with minimum area $\mathcal{A}\left(\operatorname{conv}\left(X_{n}+\rho K\right)\right)$ and maximum parametric density, respectively.

Problem 2 (Minimum Perimeter Problem). Determine packings $X_{n}+K$ with minimum perimeter of their convex hull and of the polygon $\operatorname{conv}\left(X_{n}\right)$, respectively.

Problem 3 (Minimum Container Problem). Determine packings $X_{n}+K$ with a minimum circumscribed circle or minimum dilatate of another container $K^{\prime} \in \mathcal{K}^{2}$.

Problem 4 (Minimum Diameter Problem). Determine packings $X_{n}+K$ with minimum diameter

$$
\max \left\{\left\|x-x^{\prime}\right\| \mid x, x^{\prime} \in X_{n}\right\}
$$

Besides these problems there are many others fitting in our scheme. For example, Problems 1 and 2 are special cases of the minimization of arbitrary monotone valuations on the family

$$
\mathcal{P}_{K}(n)=\left\{\operatorname{conv}\left(X_{n}\right) \mid X_{n}+K \text { is a } K \text {-packing }\right\}
$$

of packing polygons. This generalization of known packing problems is studied by the author in [S1] and includes the minimization of certain "invariant measures" as considered by Graham et al. [GWZ]. Of course, we are not able to deal with all finite packing problems, as the minimization of the "second moment" (see [GS]).

In many cases one might conjecture that solutions to the problems are lattice packings, supported by the fact that the solutions converge to lattice packings, if $n$ becomes large (see $[\mathrm{BE}],[\mathrm{F}]$, and [S1]). A result by Graham et al. [GLNÖ] indicates that in many cases solutions to the minimum container problem with unit disks in a circle are non-lattice packings. For the minimum diameter problem Erdös [E] conjectured that the minimum is not attained by a (hexagonal) lattice packing for all sufficiently large $n .^{1}$

In this paper we prove Erdös' conjecture. Moreover, we show that lattice packings $X_{n}+K$, where the polygon $\operatorname{conv}\left(X_{n}\right)$ has certain properties (see Lemma 8), are not solutions to any of the considered problems. As a consequence we find (see Theorem 9):

Extremal (best) packings $X_{n}+K$ are non-lattice packings for all sufficiently large $n$, in Problem 1 with a sufficiently large parameter $\rho$, and in Problem 2-4 in general.

The next section gives a detailed description of the attained results. The proofs are in Section 4. In Section 3 we provide some additional information about packings with unit disks.

\footnotetext{
${ }^{1}$ From Erdös' letter: "Vielleicht kann man zeigen, daß für $n>n_{0} x_{1}, \ldots, x_{n}$ nie im Dreiecksgitter einbettbar ist (aber soweit ich weiß wurde dies niemals gezeigt) ..."
} 


\section{Results}

We consider some common features of the problems listed in the Introduction: First, we may restrict ourselves to packings $X+K$ with $K \in \mathcal{K}_{o}^{2}$, where $\mathcal{K}_{o}^{2}$ is the family of centrally symmetric sets in $\mathcal{K}^{2}$ with the origin $o$ as center. This is due to the fact that $X+K$ is a packing if and only if $X+\frac{1}{2}(K+(-K))$ is one (see [M]), and the difference body $\frac{1}{2}(K+(-K))$ of $K$ is centrally symmetric and strictly convex.

With every finite packing $X_{n}+K$ we associate the convex center-polygon $\operatorname{conv}\left(X_{n}\right)$. Let $\mathcal{P}$ denote the set of all convex polygons and convex hulls of finite point sets, respectively. Then, given $n \in \mathbb{N}$ and $K \in \mathcal{K}_{o}^{2}$, in each of the problems listed in the Introduction one has to minimize a real-valued function $f: \mathcal{P} \mapsto \mathbb{R}$ on a subset $\mathcal{P}_{K}(n)$ of $\mathcal{P}$.

Example 1. Because

$$
\operatorname{conv}\left(X_{n}\right)+\rho K=\operatorname{conv}\left(X_{n}+\rho K\right)
$$

the function

$$
f(P)=\mathcal{A}(P+\rho K)
$$

with $P=\operatorname{conv}\left(X_{n}\right)$ is such a function for the maximum parametric density problem. The area of $\operatorname{conv}\left(X_{n}+\rho K\right)$, and hence $f(P)$, evaluates by Steiner's formula to

$$
f(P)=\mathcal{A}(P)+\rho \cdot 2 \mathcal{A}(P, K)+\rho^{2} \cdot \mathcal{A}(K),
$$

where $\mathcal{A}(\cdot, \cdot)$ denotes the mixed area of two convex disks (see $[\mathrm{Sch}])$. Note that

$$
f(P)=2 \mathcal{A}(P, D)=\mathcal{A}(P, 2 D),
$$

with the unit disk $D$, is the perimeter function used for the minimum perimeter problem. For the minimum container problem we consider

$$
f(P)=\min \left\{\lambda \mid P+K \subset t+\lambda K^{\prime} \text { for a } t \in \mathbb{E}^{2}\right\}
$$

for a given container $K^{\prime} \in \mathcal{K}^{2}$. The natural choice for the minimum diameter problem is, of course, the diameter function itself.

All functions $f$ in the example are independent of the position of the packing. Moreover, if one packing polygon $P_{1}$ is contained in the relative interior relint $\left(P_{2}\right)$ of another packing polygon $P_{2}$, the value of $f$ is smaller and the packing is "better" with respect to the given problem. Thus we are led to

Definition 2. A function $f: \mathcal{P} \mapsto \mathbb{R}$ is a packing function if it is

1. invariant with respect to translations, that is,

$$
f(P)=f(P+t) \quad \text { for all } \quad P \in \mathcal{P} \quad \text { and } \quad t \in \mathbb{E}^{2},
$$


2. monotonically increasing with respect to inclusion, that is,

$$
f\left(P_{1}\right) \leq f\left(P_{2}\right) \quad \text { for } \quad P_{1}, P_{2} \in \mathcal{P} \quad \text { with } \quad P_{1} \subset P_{2},
$$

moreover,

$$
f\left(P_{1}\right)<f\left(P_{2}\right), \quad \text { if } \quad P_{1} \subset \operatorname{relint}\left(P_{2}\right) .
$$

Given $K \in \mathcal{K}_{o}^{2}$ and $n \in \mathbb{N}, X_{n}+K$ is an extremal packing with respect to $f$ if $\operatorname{conv}\left(X_{n}\right)$ minimizes $f$ within $\mathcal{P}_{K}(n)$.

Note that for some packing functions $f$, there are no extremal packings, since the minimum may not exist. It is attained, though, if $f$ is continuous on $\mathcal{P}$, which is turned into a metric space with the Hausdorff-metric (see [Sch]). There are, of course, many more packing functions than the ones considered in Example 1. We may in particular generalize the problems listed in the Introduction as follows.

Example 3. In (1), hence for the maximum parametric density problem, the packing function depends on the disk $K$ of the packing. More generally, we may consider a packing function $f$ with

$$
f(P)=\mathcal{A}\left(P+\rho K^{\prime}\right),
$$

where still $P=\operatorname{conv}\left(X_{n}\right)$ and $X_{n}+K$ is a finite packing of $K$, and $K^{\prime} \in \mathcal{K}^{2}$ is another disk, independent of $K$. Similarly, (3) for the minimum perimeter problem may be replaced by the more general

$$
f(P)=\mathcal{A}\left(P, K^{\prime}\right)
$$

The minimum diameter problem may be generalized by replacing the Euclidian norm distance by the $K^{\prime}$-distance, for some $K^{\prime} \in \mathcal{K}_{o}^{2}$. For two arbitrary sets $X, X^{\prime} \subset \mathbb{E}^{2}$ the $K^{\prime}$-distance is given by

$$
\inf \left\{\lambda \geq 0 \mid x-x^{\prime} \in \lambda K^{\prime}, x \in X, x^{\prime} \in X^{\prime}\right\},
$$

and therefore we may replace the diameter function by the more general packing function

$$
f(P)=\max \left\{\lambda \mid x-x^{\prime} \in \lambda K^{\prime}, x, x^{\prime} \in X_{n}\right\} .
$$

It is easily checked that the packing functions in the example are continuous. Hence, we are led to the following:

Remark. Let $K \in \mathcal{K}^{2}$ and $f: \mathcal{P} \mapsto \mathbb{R}$ be one of the packing functions in (4), (6), (8) or (5). Then for every $n \in \mathbb{N}$ there exists an extremal packing $X_{n}+K$.

Given a disk $K \in \mathcal{K}_{o}^{2}$ and a packing function $f$, we want to know if there exist extremal lattice packings $X_{n}+K$. Because of the monotonicity of $f$ we may assume that an extremal lattice packing $X_{n}+K$ is saturated, that is, $\operatorname{conv}\left(X_{n}\right) \cap \Lambda=X_{n}$ for a packing lattice $\Lambda$ of $K$. Thus it suffices to consider the set of lattice- or $\Lambda$-polygons $\operatorname{conv}\left(X_{n}\right) \in \mathcal{P}_{K}(n)$ 
By the definition, a finite lattice packing $X_{n}+K$ is not extremal with respect to any packing function if there exists a packing $X_{n}^{\prime}+K$ with

$$
\operatorname{conv}\left(X_{n}^{\prime}\right) \subset \operatorname{relint}\left(\operatorname{conv}\left(X_{n}\right)\right) .
$$

With this argument many lattice packings can be excluded. Let

$$
\lambda_{i}(\Lambda, K)=\min \{\lambda \mid \operatorname{dim}(\lambda K \cap \Lambda) \geq i\}
$$

denote the $i$ th successive minimum of a lattice $\Lambda$ and $K \in \mathcal{K}_{o}^{2}$. Here, $\operatorname{dim}(\cdot)$ denotes the dimension (of the affine hull) of a set in $\mathbb{E}^{2}$. The definition implies $\lambda_{2}(\Lambda, K) \geq \lambda_{1}(\Lambda, K)$ and $\Lambda+K$ is a packing if and only if $\lambda_{1}(\Lambda, K) \geq 2$. If $\Lambda$ is a packing lattice with $\lambda_{2}(\Lambda, K)=2$, then $\Lambda$ is characterized by the fact that every translate of $K$ in $\Lambda+K$ meets at least four others. We show

Lemma 4. Let $K \in \mathcal{K}_{o}^{2}$ and $X_{n}+K$ be a lattice packing that is extremal with respect to a packing function. Then there exists a packing lattice $\Lambda$ with $X_{n} \subset \Lambda$ and either

1. $\lambda_{2}(\Lambda, K)=2$

or

2. $\lambda_{2}(\Lambda, K)>\lambda_{1}(\Lambda, K)=2$ and $X_{n}$ is in between two parallel lines of $K$-distance 2 (see (7)) which both intersect $X_{n}$ in exactly one point.

Case 2 is superfluous if $f$ is a packing function as in (5) or (6).

In the second case the packing and the packing polygon respectively is two-dimensional, but "flat". This case occurs in the minimum container problem, with a suitable container $K^{\prime}$.

Example 5. We consider a packing with three copies of the unit disk $D$, where the centers form an isosceles triangle $T$ with base length 2 and height $h$, with $\sqrt{3}<h<$ $\sqrt{5+2 \sqrt{5}}$ (see Fig. 1).

If we consider $f$ as in (4), where the container $K^{\prime}$ is the convex hull of the packing, then $f(T)=1$ is the minimum of $f$ for all "packing triangles", and it is attained only by triangles with the same base as $T$ and height between $\sqrt{3}$ and $h$. Thus the minimum is attained by lattice packings with respect to lattices $\Lambda$ with $\lambda_{2}(\Lambda, D)>2$ as well as such with $\lambda_{2}(\Lambda, D)=2$.

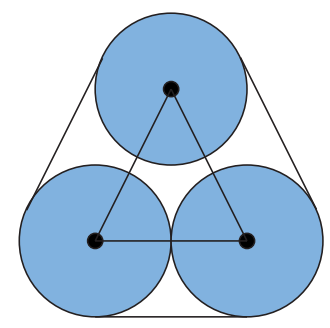

Fig. 1. Example for the necessity of case 2 in Lemma 4. 
Next, we take a closer look at possible lattice polygons $P$ with respect to a fixed lattice $\Lambda$. Here, because of Lemma 4 , in particular the case $\lambda_{2}(\Lambda, K)=2$ is of interest. We show that certain polygons and packings respectively are locally and even globally "unstable" (improvable) with respect to any packing function. To characterize these, we consider the (primitive) edges of $P$, which are segments $[x y] \subset \operatorname{bd}(P)$ satisfying $[x y] \cap \Lambda=\{x, y\}$.

Definition 6. An edge $[x y]$ of a $\Lambda$-polygon $P$ is called loose with respect to $K \in \mathcal{K}_{o}^{2}$, if all $z \in \Lambda$ satisfy

$$
(z+K) \cap(x+K)=\emptyset \quad \text { or } \quad(z+K) \cap(y+K)=\emptyset .
$$

Thus edges exceeding a certain length are loose, whereas edges $[x y]$ with $(x+K) \cap$ $(y+K) \neq \emptyset$ are not.

To state a sufficient condition for the global instability of a lattice packing, we define the neighbors of a lattice point with respect to a $K \in \mathcal{K}_{o}^{2}$ as the set of all lattice points with $K$-distance 2 . It was already noticed by Minkowski [M] that points of a packing lattice have at most six neighbors. Moreover, the number of neighbors is divisible by 2 because of $K$ 's symmetry. Thus in a packing lattice with $\lambda_{2}(\Lambda, K)=2$ the lattice points have four or six neighbors.

Definition 7. We say that the vertex $x$ of a lattice polygon $P$ is obtuse if there is a neighbor $y$ of $x$, such that the neighbors of $y$ are all in $P$.

We show

Lemma 8. Let $K \in \mathcal{K}_{o}^{2}$ and $X_{n}+K$ be a lattice packing such that $\operatorname{conv}\left(X_{n}\right)$ has at least one loose edge and only obtuse vertices. Then $X_{n}+K$ is not extremal with respect to any packing function.

Thus Lemma 8 gives us a geometrical criterion to recognize non-extremal lattice packings. For the problems listed in the Introduction and their generalizations described in Example 3 we derive

Theorem 9. Let $K \in \mathcal{K}^{2}$ and $f: \mathcal{P} \mapsto \mathbb{R}$ be one of the packing functions in (4), (6), (8) or (5) with sufficiently large parameter $\rho$. Then extremal packings $X_{n}+K$ with respect to $f$ are non-lattice packings for all sufficiently large $n$.

Note that the packing functions in Theorem 9 all depend on a $K^{\prime} \in \mathcal{K}^{2}$. For $f$ with $f(P)=\mathcal{A}\left(P+\rho K^{\prime}\right)$ as in (5), we are able to give a bound $\rho\left(K, K^{\prime}\right)$ such that all parameters $\rho$ with $\rho>\rho\left(K, K^{\prime}\right)$ are "sufficiently large". The parameter $\rho\left(K, K^{\prime}\right)$ and ways to determine it are studied by the author in [S1]. For the unit disk $D$ we obtain

$$
\rho(D, D)=\frac{\sqrt{3}}{2(1+\sqrt{3}-\sqrt{7})}=10.035 \ldots
$$



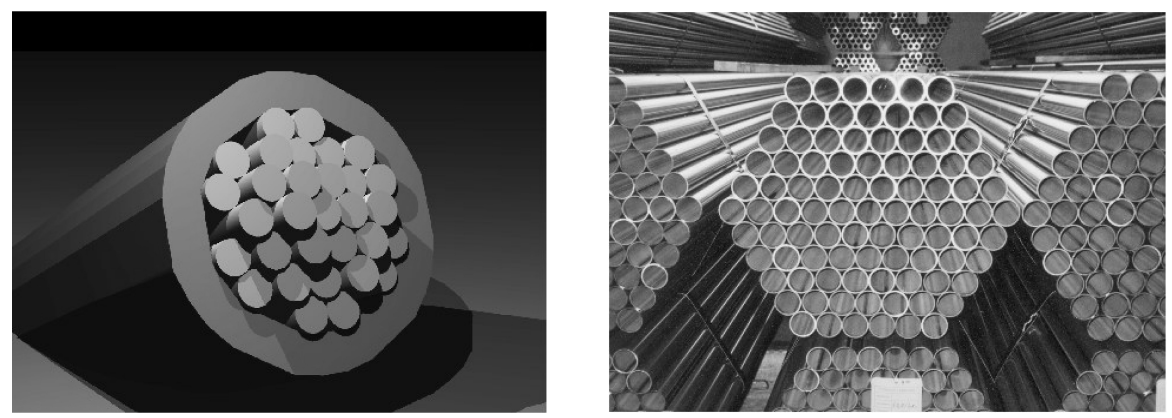

Fig. 2. Hexagonal packings in a multicore cable and a pipe bundle.

\section{Packings with Unit Disks}

In this section we discuss some details of packings with unit disks, and hereby give some illustrations of results and proofs. We consider, in particular, the minimum perimeter problem for which, besides an asymptotic result (see [Z]), surprisingly little is known in comparison with other packing problems, although there are obvious practical problems, such as the minimization of material needed to insulate a multicore cable or to tie up a collection of pipes (see Fig. 2). Besides, the question occurs in other mathematical contexts, such as in the determination of a minimum rope length of knots and links (see $[\mathrm{CKS}]$ ).

Nevertheless, there are a few interesting phenomena which are worth mentioning in our view. For a fixed $n$, solutions to the perimeter problem are attained by minimizing $\mathcal{A}(P+\rho D)$ for a sufficiently large parameter $\rho$ (see [S1]). The close relation of the two problems becomes clear by Steiner's formula (2).

By Theorem 9 and (9) there exists $n_{0} \in \mathbb{N}$, depending on $\rho>10.035 \ldots$, such that extremal packings $X_{n}+D$ with respect to $f(P)=\mathcal{A}(P+\rho D)$ are non-lattice packings for all $n \geq n_{0}$. On the other hand, for smaller $n$ and smaller $\rho$, lattice packings are extremal.

We first look at smaller parameters: It follows from results of Wegner [W] and Groemer [G] that, despite some exceptions,

$$
f(P)=\mathcal{A}(P+\rho D) \quad \text { with } \quad \rho \leq 1
$$

is minimized by Groemerpackings (see [S2]). These are lattice packings with respect to a hexagonal lattice $\Lambda$, which is characterized by the fact that every disk in the packing $\Lambda+D$ touches six others (see, e.g. Fig. 2). The center-polygon of a Groemerpacking has only edges of length 2 and therefore only non-loose edges. In the still open exceptional cases, extremal packings are conjectured to be hexagonal lattice packings with their center polygons having non-loose edges only as well (see [S2]).

These results incite us to investigate the gap of parameters between 1 and $10.035 \ldots$ A result in this direction is a refinement of Lemma 4 for the parametric density problem with unit disks. We show in [S2] that only hexagonal lattice packings minimize $f$ within 

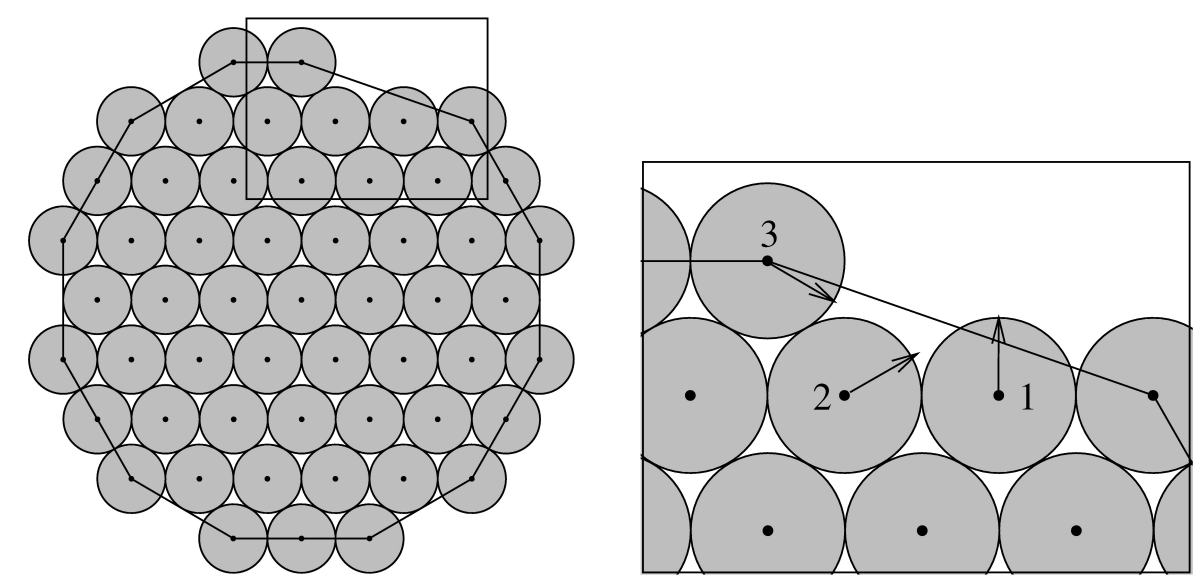

Fig. 3. Lattice packing with minimum perimeter, which is improvable by moving three disks successively.

the set of lattice packings $X_{n}+D$, hence

extremal lattice packings are necessarily hexagonal.

This is especially interesting, since a corresponding result for general $K \in \mathcal{K}^{2}$ is not possible (see [S1]). For the perimeter problem it implies that within the set of lattice solutions there is a hexagonal packing. In contrast to the parametric density problem these are not necessarily the only solutions. For example, the minimum perimeter of a packing with four unit disks is attained in all lattices $\Lambda$ with $\Lambda_{2}(\Lambda, D)=2$.

Thus for both problems the determination of a best lattice packing is reduced to the comparison of finitely many hexagonal packings. For an efficient search, a unique description of lattice polygons with respect to a fixed lattice is developed by the author in [S1]. Calculations based on this method yield that the packing shown in Fig. 3 attains the minimum perimeter and the maximum parametric density for parameters $\rho>21.911 \ldots$, within the family of lattice packings with 54 unit disks.

As indicated in Fig. 3, it is possible to move three translates successively to attain a non-lattice packing with smaller perimeter. Moreover, as in the proof of Lemma 8, we are able to move all translates of the packing and find a packing with smaller diameter and smaller circumscribed circle as well.

Moreover, our calculations show that 54 is the smallest $n$ for which the center-polygon of a best lattice packing has a loose edge. So we are led to

Conjecture 10. The minimum perimeter of a packing with $n$ unit disks is attained by (hexagonal) lattice packings for all $n \leq 53$.

Note that there may also be extremal non-lattice packings such as extremal packings with five and six translates. For sufficiently many translates, lattice packings are not extremal by Theorem 9. With the techniques developed in [S1] we are in particular able 
to show:

For $n \geq 371$ only non-lattice packings $X_{n}+D$ are extremal with respect to the perimeter function.

In the light of this result, it is even more remarkable that extremal packings converge to hexagonal packings, if $n$ becomes large (see [Z]).

\section{Proofs}

Proof of Lemma 4. Let $X_{n}+K$ be an extremal packing with respect to $f$ and let $\Lambda$ be a lattice with $X_{n} \subset \Lambda$. If $\lambda_{1}(\Lambda, K)>2$, then $X_{n}+\lambda K$ with $1<\lambda \leq \lambda_{1}(\Lambda, K) / 2$ is a packing, as well as $(1 / \lambda) X_{n}+K$, and we find a suitable $t \in \mathbb{E}^{2}$ with $t+(1 / \lambda) X_{n} \in$ $\operatorname{relint}\left(\operatorname{conv}\left(X_{n}\right)\right)$. Thus we may assume $\lambda_{1}(\Lambda, K)=2$.

If $\lambda_{2}(\Lambda, K)=2$, then condition 1 is valid. So we assume there is no lattice $\Lambda$ with $X_{n} \subset \Lambda$ and $\lambda_{2}(\Lambda, K)=2$. Then $\operatorname{conv}\left(X_{n}\right)$ is not a segment. Because $\lambda_{1}(\Lambda, K)=2$, the translates of $X_{n}+K$ can all be moved in between parallel tangents of $K$. This can be done without overlappings, and such that no translate touches any other afterwards. Thus in a second step the translates can be moved in any direction to form a new packing $X_{n}^{\prime}+K$.

In this process every $x \in X_{n}$ can be moved to an $x^{\prime} \in \operatorname{int}\left(\operatorname{conv}\left(X_{n}\right)\right)$, except if the parallel of the tangents through $x$ meets $\operatorname{conv}\left(X_{n}\right)$ only in $x$. Thus there are at most two of these points, say $x_{1}, x_{2}$, and $X_{n}$ lies between the corresponding tangents through $x_{1}$ and $x_{2}$. If $x_{1}+K$ and $x_{2}+K$ do not touch each other, then they can be moved without overlapping to points $x_{1}^{\prime}, x_{2}^{\prime} \in \operatorname{int}\left(\operatorname{conv}\left(X_{n}\right)\right)$, after the other translates have been moved. If, on the other hand, $x_{1}+K$ and $x_{2}+K$ do touch each other, then we have found a packing satisfying condition 2 of the lemma.

For a packing function as in (5) or (6), condition 2 in Definition 2 may be extended by

$$
f\left(P_{1}\right)<f\left(P_{2}\right), \quad \text { if } \quad P_{1} \subset P_{2} \quad \text { and } \quad P_{1} \neq P_{2} .
$$

Thus in case 2 we may move the vertices of $\operatorname{conv}\left(X_{n}\right)$ not lying on the described parallels into the interior of $\operatorname{conv}\left(X_{n}\right)$ and obtain a better packing, which is not possible if it is extremal.

Proof of Lemma 8. Let $\Lambda \subset \mathbb{E}^{2}$ be a packing lattice of a $K \in \mathcal{K}_{o}^{2}$. Further, we choose $X_{n} \subset \Lambda$ such that $P=\operatorname{conv}\left(X_{n}\right)$ has at least one loose edge and only obtuse vertices with respect to $K$.

First we assume $\lambda_{2}(\Lambda, K)>2$. Then by Lemma 4 we know $X_{n}+K$ is not extremal with respect to any packing function or $\lambda_{1}(\Lambda, K)=2$ and $X_{n}$ is in between two parallel lines of $K$-distance 2 which both intersect $X_{n}$ in exactly one point. Then $P$ has a nonobtuse vertex, which contradicts the assumptions on $P$. So we may assume $\lambda_{2}(\Lambda, K)=2$ and $\operatorname{int}(P) \neq \emptyset$ because a segment $P$ has two non-obtuse vertices, if $\lambda_{2}(\Lambda, K)=2$. Besides, by our looseness assumption we would find a better packing segment (sausage) contained in $\operatorname{relint}(P)$. 
Because $\lambda_{2}(\Lambda, K)=2$ there exists a base $a_{1}, a_{2} \in \Lambda \cap 2 K$ of $\Lambda$ (see [GL]), that is,

$$
\Lambda=\left\{u_{1} a_{1}+u_{2} a_{2} \mid u_{1}, u_{2} \in \mathbb{Z}\right\} .
$$

Moreover, if a lattice point $x \in \Lambda$ has six neighbors with respect to $K$, we may assume that the neighbors are

$$
\left\{x+y \mid y \in\left\{ \pm a_{1}, \pm a_{2}, \pm\left(a_{2}-a_{1}\right)\right\}\right\} .
$$

Since a linear transformation keeps information on neighborhood, obtuseness and looseness, we may think of $\Lambda$ as

I. the integral lattice $\mathbb{Z}^{2}$, or

II. a hexagonal lattice, e.g. with base $a_{1}=(1,0), a_{2}=\left(\frac{1}{2}, \sqrt{3} / 2\right)$.

Note that in case I, loose edges are those of length $\geq \sqrt{5}$, and in case II, edges of length $\geq \sqrt{7}$. Moreover, non-loose edges are of length 1 or $\sqrt{2}$ in case I and of length 1 or $\sqrt{3}$ in case II.

Given a lattice packing of type I or II, we want to describe a way of sequentially moving translates to obtain a better non-lattice packing. In this process we only allow movements of translates $x+K, x \in X_{n}$, to translates $x^{\prime}+K, x^{\prime} \notin \Lambda$, such that $x^{\prime}+K$ does not touch any other translate. Under this essential precondition we use the following terminology:

A lattice point $x \in \Lambda$ is called free if there is no translate $x+K$. Thus starting with a lattice packing $X_{n}+K$, the corresponding set of free lattice points is $\Lambda \backslash X_{n}$. Moreover, if we move a translate $x+K$ as described above, then $x \in X_{n}$ is free afterwards.

We call a lattice point movable, if it has one free neighbor in case I and two free adjacent neighbors in case II. Hence, if $x \in X_{n}$ is movable, then it is possible to move $x+K$ in the required way in between two parallel tangents of $x+K$. Hence $x$ is free afterwards. Moreover, if we sequentially free movable lattice points of $X_{n}$ in this way, then after every step there are possible new movable lattice points, because the number of disk contacts decreases. The assertion follows, if we show:

(a) There is either a movable lattice point in $\operatorname{int}(P)$, or all interior lattice points of $P$ are free.

(b) If all interior lattice points of $P$ are free, then the lattice points in $\operatorname{bd}(P)$ are sequentially movable into $\operatorname{int}(P)$.

In order to see how movable lattice points are moved into or within the interior, it is important to know in which direction a translate $x+K$ is moved in between the parallel tangents. Without loss of generality the tangents run through $x \pm \frac{1}{2} a_{1}$. Because of $\lambda_{2}(\Lambda, K)=2$ we know that $x+K$ contains the quadrilateral

$$
x+\frac{1}{2} \cdot \operatorname{conv}\left\{ \pm a_{1}, \pm a_{2}\right\}
$$

in case I, and the hexagon

$$
x+\frac{1}{2} \cdot \operatorname{conv}\left\{ \pm a_{1}, \pm a_{2}, \pm\left(a_{2}-a_{1}\right)\right\}
$$

in case II (see Fig. 4). 

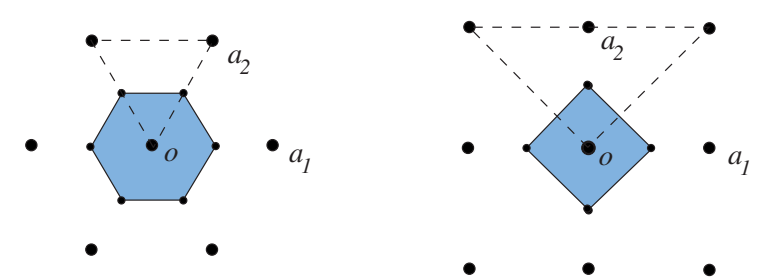

Fig. 4. In both cases $K$ can be moved without overlappings and with its center remaining in the dashed region.

Since $K$ is strictly convex the tangents of $K$ through $x \pm \frac{1}{2} a_{1}$ meet the quadrilateral and hexagon respectively only in $x \pm \frac{1}{2} a_{1}$. We may further assume that $x+a_{2}$ is free in case I, and that $x+\left(a_{2}-a_{1}\right)$ is additionally free in case II. Then there exists a point $x^{\prime}$ in the interior of

$$
x+\operatorname{conv}\left\{o,\left(a_{2}+a_{1}\right),\left(a_{2}-a_{1}\right)\right\}
$$

in case $\mathrm{I}$, and in the interior of

$$
x+\operatorname{conv}\left\{o, a_{2},\left(a_{2}-a_{1}\right)\right\}
$$

in case II, such that $x+K$ can be moved to $x^{\prime}+K$ without overlapping.

This already allows us to verify (b): We assume that the lattice points in int $(P)$ are all free and let $x$ be a lattice point in $\operatorname{bd}(P)$. Then, by the assumptions of the lemma that all vertices are obtuse, we find a neighbor $y \in \operatorname{int}(P)$ with all neighbors of $y$ being in $P$. This is clear by Definition 7 if $x$ is a vertex of $P$. If $x$ is not a vertex of $P$, there are two vertices $x_{1}$ and $x_{2}$ of $P$ with $x \in\left[x_{1} x_{2}\right]$. By our assumption, $x_{1}$ and $x_{2}$ have neighbors $y_{1}$ and $y_{2}$ with their neighbors being in $P$. Thus the convex hull of these neighbors are also in $P$ and we find $y$ as asserted.

Therefore $x$ can be moved into $\operatorname{int}(P)$ in case I, and in case II, if one of the two neighbors common to $x$ and $y$ is free. If both neighbors are not, then both are necessarily in $\operatorname{bd}(P)$ and one of them can be freed before $x$. Since otherwise $\operatorname{bd}(P)$ would be a hexagon without any loose edge, which contradicts the assumption on $P$. Hence, this proves (b) and it remains to verify (a).

For this, let $[y z]$ be a loose edge of $P$. Since $P$ is no segment, there is a lattice point $x \in P \cap \Lambda$ such that the triangle $[x y z]$ is in $P$ and the projection of $x$ onto the line through $y$ and $z$ lies in $[y z]$. We may further assume that $x$ is in the interior of $P$, because otherwise $P$ has a non-obtuse vertex, against our assumption on $P$.

Since $[y z]$ is loose, we find that $x$ has a free neighbor in case I and two free adjacent neighbors in case II, which are, in each case, on the opposite side of the line through $y$ and $z$. This is clear, if neither $y$ nor $z$ are neighbors of $x$. If $y$ and $z$ are both neighbors of $x$, then $x+K$ touches both $y+K$ and $z+K$, in contradiction to the definition of a loose edge. If one of them is a neighbor, say $y$, then $z$ lies on the parallel of the line through $y$ and $x$, which runs through one other neighbor of $x$ in case $\mathrm{I}$, and two other neighbors of $x$ in case II. Thus in each case these neighbors are not in $P$ and therefore they are free. As a consequence, $x$ is movable within the interior of $P$. Moreover, in case II we find at least one neighbor of $x$ in $P$, which shares one of the two free neighbors with $x$. 

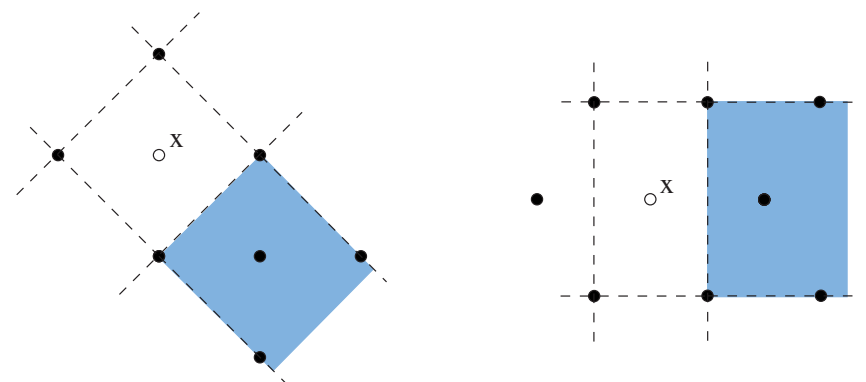

Fig. 5

Otherwise we could find a non-obtuse vertex of $P$. Thus (a), and therefore the lemma, follows if we show that every lattice point in $\operatorname{int}(P)$ has one interior or free neighbor in case I, and two interior or free neighbors in case II.

So we assume in case $\mathrm{I}$ there is no interior or free neighbor of $x \in \operatorname{int}(P)$. Then all four neighbors of $x$ lie in $\operatorname{bd}(P)$. The four lines through adjacent neighbors of $x$ all separate $x$ from a possibly empty part of $P$. These four parts each lie between two parallels of the four lines (see Fig. 5). Therefore we find a non-obtuse vertex or no loose edge in each of these parts, which is not possible by our assumption on $P$.

In case II the situation is a bit different: if we assume that there are no two, interior or free adjacent neighbors of $x \in \operatorname{int}(P)$, then at least every second neighbor is in $\operatorname{bd}(P)$. We distinguish two cases:

If all other neighbors of $x$ are in $P$ too, then we consider the six lines through adjacent neighbors of $x$. Two parallels of these lines run through four neighbors, and the two parallel, orthogonal lines through these four neighbors separate $x$ and two parts of $P$, which lie between the other parallels (see Fig. 5). Here, as in case I, we find a non-obtuse vertex or no loose edge in each part, which is not possible.

It remains to look at the case when at least one neighbor $y$ of $x$ does not belong to $P$. Then the segment between the two neighbors common to $x$ and $y$ are in $\operatorname{bd}(P)$. Moreover, $2 x-y \in \operatorname{bd}(P)$ and the parallel lines containing the segment and $2 x-y$ enclose a stripe with every lattice point in it having a neighbor outside. The line through $x$ and $y$ cuts $P$ into two pieces, one of them contained in the described stripe (see Fig. 6). Thus in this part of $P$ we find a non-obtuse vertex, in contradiction to our assumptions on $P$.

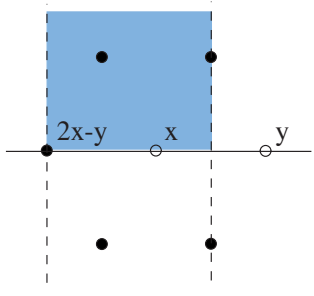

Fig. 6 
Proof of Theorem 9. By Lemma 8, the theorem is true, if we show that in all cases best lattice packings have center-polygons with at least one loose edge and only obtuse vertices for all sufficiently large $n$. We show this by looking at the asymptotic shape of best lattice packings. To put this in concrete terms, let

$$
R(M)=\min \left\{R>0 \mid M \subset t+R \cdot D, t \in \mathbb{E}^{2}\right\}
$$

denote the circumradius of a compact set $M \subset \mathbb{E}^{2}$ with $\operatorname{int}(M) \neq \emptyset$ and $c(M)$ the center of its circum circle. Hence $M \subset c(M)+R(M) \cdot D$. The shape of $M$ is defined by

$$
\mathcal{S}(M)=(\operatorname{conv}(M)-c(M)) / R(M) .
$$

The shapes of best or extremal (lattice) packings $\left\{X_{n}+K\right\}_{n \in \mathbb{N}}$ (and their centerpolygons $\operatorname{conv}\left(X_{n}\right)$, respectively) with respect to one of the packing functions in (4), (6) or (8) converge to the shape of $K^{\prime}$. That is,

$$
\lim _{n \rightarrow \infty} \mathcal{S}\left(\operatorname{conv}\left(X_{n}\right)\right)=\mathcal{S}\left(K^{\prime}\right),
$$

where the family of compact subsets in $\mathbb{E}^{d}$ is made into a metric space with the Hausdorffmetric (see [Sch]). This is easily seen for the packing functions in (4) and (8) by rearranging translates in a hypothetical subsequence not satisfying (10). For packing functions as in (6), equation (10) is the two-dimensional case of the result in [S3].

For a packing function as in (5), the situation is a bit more complicated: By applying a theorem of Wulff, we show in [S1] for a given lattice $\Lambda$ that the shapes of best $\Lambda$-packings $\left\{X_{n}+K\right\}_{n \in \mathbb{N}}$ converge to the shape of a $\Lambda$-polygon $P_{\rho}$, provided that the parameter $\rho$ in (5) is sufficiently large. Hence

$$
\lim _{n \rightarrow \infty} \mathcal{S}\left(\operatorname{conv}\left(X_{n}\right)\right)=\mathcal{S}\left(P_{\rho}\right) .
$$

Moreover, the shapes of these polygons converge to $K^{\prime}$ for growing parameters, hence

$$
\lim _{\rho \rightarrow \infty} \mathcal{S}\left(P_{\rho}\right)=\mathcal{S}\left(K^{\prime}\right) .
$$

Thus there exists a parameter $\rho_{0}(\Lambda)$ such that $P_{\rho}$ with $\rho \geq \rho_{0}(\Lambda)$ has at least one loose edge and only obtuse vertices with respect to $\Lambda$.

Now, the space of lattices can be made into a topological space (see [GL]) and we find that $\rho_{0}$ is a continuous function of lattices (see [S1]). By Lemma 4 it suffices to consider packings with respect to lattices in the compact family

$$
\left\{\Lambda \mid \lambda_{1}(\Lambda, K)=\lambda_{2}(\Lambda, K)=2\right\},
$$

which implies that $\rho_{0}$ attains its maximum. Thus for all larger parameters the centerpolygons conv $\left(X_{n}\right)$ of best or extremal lattice packings $X_{n}+K$ have loose edges and only non-obtuse vertices for all sufficiently large $n$.

\section{Final Remarks}

Throughout the whole paper we consider only strictly convex $K$ and $K^{\prime}$, respectively. From case to case, but not generally, analogical results hold for a wider class of compact 
convex disks. For example, solutions to the container problem with a non-strictly convex container may as well be non-lattice packings for all sufficiently large $n$, as packings of unit disks into a regular $k$-gon with $k>12$, since there are at most 12 different types of non-loose edges with respect to a lattice $\Lambda$. This follows from the proof of Lemma 8 .

In the mentioned proof we distinguish two types of lattice packings of a given $K \in \mathcal{K}_{o}^{2}$-one with four, the other with six neighbors of each translate. The latter one, say the "hexagonal type", is of particular importance for the maximum parametric density problem and its generalizations in (5). In [S1] we show that for sufficiently large $n$, best or extremal lattice packings are always of this hexagonal type. This is in a way a finite analogue of a well-known result of Swinnerton-Dyer on densest packing lattices (see [GL]).

\section{Acknowledgment}

I thank the referees for their valuable comments and especially Peter Brass for pointing me to the problem by Paul Erdös and for sending me a copy of his letter [E].

\section{References}

[BE] Paul Bateman and Paul Erdös, Geometrical Extrema Suggested by a Lemma of Besicovitch, Amer. Math. Monthly, 58 (1951), 306-314.

[BHW] Ulrich Betke, Martin Henk and Jörg M. Wills, Finite and Infinite Packings, J. Reine Angew. Math., 453 (1994), 165-191.

[BF] András Bezdek and Ferenc Fodor, Minimal Diameter of Certain Sets in the Plane, J. Combin. Theory Ser. A, 85 (1999), 105-111.

[CKS] Jason Cantarella, Robert B. Knuser and John M. Sullivan, On the Minimum Ropelength of Knots and Links, Invent. Math., in press.

[CS] John H. Conway and Neil J. A. Sloane, Sphere Packings, Lattices and Groups, Springer-Verlag, New York, 1998.

[CFG] Hallard T. Croft, Kenneth J. Falconer and Richard K. Guy, Unsolved Problems in Geometry, SpringerVerlag, New York, 1991.

[E] Paul Erdös, Letter to Peter Brass, 19 June 1993.

[F] László Fejes Tóth, Lagerungen in der Ebene, auf der Kugel und im Raum, 2nd improved edn., Springer-Verlag, Berlin, 1972.

[GLNÖ] Ronald L. Graham, Boris D. Lubachevsky, Kari J. Nurmela and Patric R. J. Östergård, Dense Packings of Congruent Circles in a Circle, Discrete Math., 181 (1998), 139-154.

[GS] Ronald L. Graham and Neil J. A. Sloane, Penny-Packings and Two-Dimensional codes, Discrete Comput. Geom., 5 (1990), 1-11.

[GWZ] Ronald L. Graham, Hans S. Witsenhausen and Hans J. Zassenhaus, On Tightest Packings in the Minkowski Plane, Pacific J. Math., 41 (1972), 699-715.

[GW] Peter Gritzmann and Jörg M. Wills, Finite Packing and Covering, in Handbook of Convex Geometry (Peter M. Gruber and Jörg M. Wills, eds.), North-Holland, Amsterdam, 1993, pp. 861-897.

[G] Helmut Groemer, Über die Einlagerung von Kreisen in einen konvexen Bereich, Math. Z., 73 (1960), 285-294.

[GL] Peter M. Gruber and C. Gerrit Lekkerkerker, Geometry of Numbers, North-Holland, Amsterdam, 1987.

[H] Thomas C. Hales, Sphere Packings, I, II, Discrete Comput. Geom., 17 (1997), 1-51; 18 (1997), 135-149.

[L] Jeffrey C. Lagarias, Bounds for Local Density of Sphere Packings and the Kepler Conjecture, Discrete Comput. Geom., 27 (2002), 165-193. 
[M] Hermann Minkowski, Geometrie der Zahlen, Teubner, Leipzig, 1896. Reprint, Chelsea, New York, 1953.

[Sch] Rolf Schneider, Convex Bodies: The Brunn-Minkowski Theory, Cambridge University Press, Cambridge, 1993.

[S1] Achill Schürmann, Plane Finite Packings, Shaker-Verlag, Aachen, 2000.

[S2] Achill Schürmann, On Parametric Density of Finite Circle Packings, Beiträge Algebra Geom., 41 (2000), 329-334.

[S3] Achill Schürmann, The Spherical Conjecture in Minkowski Geometry, Arch. Math., in press.

[W] Gerd Wegner, Über endliche Kreispackungen in der Ebene, Studia Sci. Math. Hungar., 21 (1986), $1-28$.

[Z] Chuanming Zong, Sphere Packings, Springer-Verlag, New York, 1999.

Received September 5, 2001, and in revised form January 24, 2002. Online publication July 10, 2002. 